PREPARED FOR THE U.S. DEPARTMENT OF ENERGY, UNDER CONTRACT DE-AC02-76CH03073

PPPL-3740

PPPL-3740

UC-70

Modeling of Neoclassical Tearing Mode Stability for Generalized Toroidal Geometry

by

A.L. Rosenberg, D.A. Gates, A. Pletzer, J.E. Menard, S.E. Kruger, C.C. Hegna, F. Paoletti, and S. Sabbagh

August 2002

$N_{\substack{\text { PRInCETOn PLASMA } \\ \text { PHYSIES LABORATORY }}}^{D}$

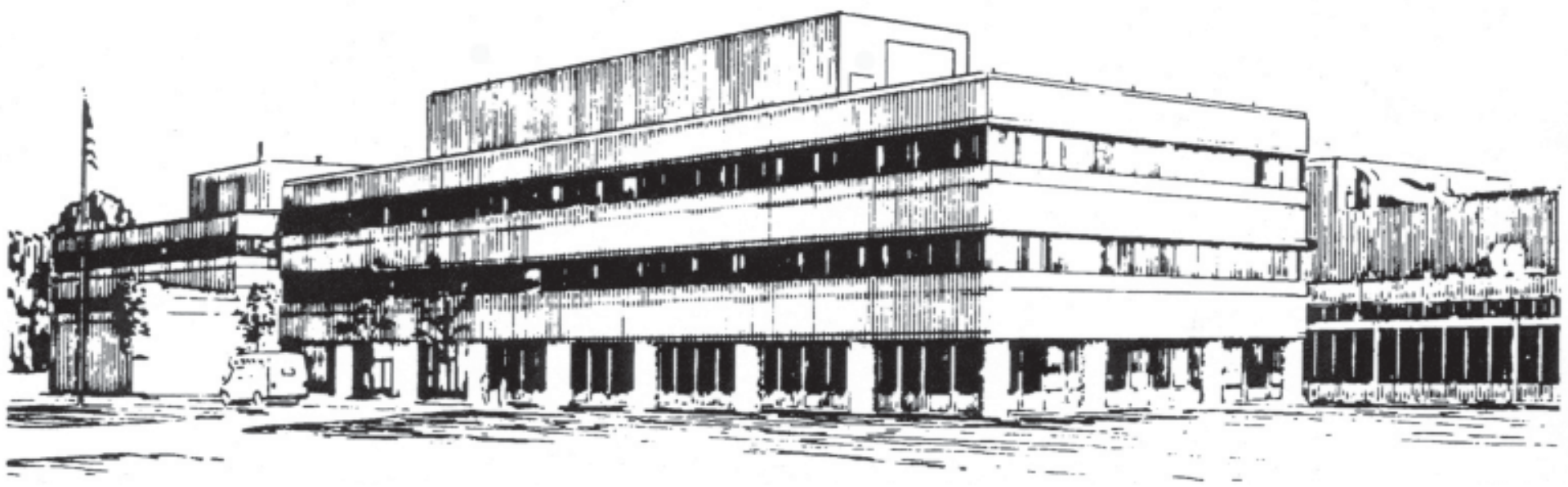

PRINCETON PLASMA PHYSICS LABORATORY PRINCETON UNIVERSITY, PRINCETON, NEW JERSEY 


\section{PPPL Reports Disclaimer}

This report was prepared as an account of work sponsored by an agency of the United States Government. Neither the United States Government nor any agency thereof, nor any of their employees, makes any warranty, express or implied, or assumes any legal liability or responsibility for the accuracy, completeness, or usefulness of any information, apparatus, product, or process disclosed, or represents that its use would not infringe privately owned rights. Reference herein to any specific commercial product, process, or service by trade name, trademark, manufacturer, or otherwise, does not necessarily constitute or imply its endorsement, recommendation, or favoring by the United States Government or any agency thereof. The views and opinions of authors expressed herein do not necessarily state or reflect those of the United States Government or any agency thereof.

\section{Availability}

This report is posted on the U.S. Department of Energy's Princeton Plasma Physics Laboratory Publications and Reports web site in Fiscal Year 2002. The home page for PPPL Reports and Publications is: http://www.pppl.gov/pub_report/

DOE and DOE Contractors can obtain copies of this report from:

U.S. Department of Energy

Office of Scientific and Technical Information

DOE Technical Information Services (DTIS)

P.O. Box 62

Oak Ridge, TN 37831

Telephone: (865) 576-8401

Fax: (865) 576-5728

Email: reports@adonis.osti.gov

This report is available to the general public from:

National Technical Information Service

U.S. Department of Commerce

5285 Port Royal Road

Springfield, VA 22161

Telephone: 1-800-553-6847 or

(703) 605-6000

Fax: (703) 321-8547

Internet: http://www.ntis.gov/ordering.htm 


\title{
Modeling of neoclassical tearing mode stability for generalized toroidal geometry
}

\author{
A. L. Rosenberg, D. A. Gates, A. Pletzer, and J. E. Menard \\ Princeton Plasma Physics Laboratory, Princeton, New Jersey 08543 \\ S. E. Kruger \\ Science Applications International Corporation, 10260 Campus Point Drive, San Diego, \\ California 92121 \\ C. C. Hegna \\ Department of Engineering Physics, Madison, Wisconsin 53706 \\ F. Paoletti and S. Sabbagh \\ Department of Applied Physics and Applied Mathematics, Columbia University, New York, \\ NY 10027
}

\begin{abstract}
Neoclassical tearing modes (NTMs) can lead to disruption and loss of confinement. Previous analysis of these modes used large aspect ratio, low $\beta$ (plasma pressure/magnetic pressure) approximations to determine the effect of NTMs on tokamak plasmas. A more accurate tool is needed to predict the onset of these instabilities. As a follow-up to recent theoretical work, a code has been written which computes the tearing mode island growth rate for arbitrary tokamak geometry. It calls PEST-3 [A. Pletzer et al., J. Comput. Phys. 115, 530 (1994)] to compute $\Delta^{\prime}$, the resistive magnetohydrodynamic (MHD) matching parameter. The code also calls the FLUXGRID routines in NIMROD [A. H. Glasser et al., Plasma Phys. Controlled Fusion 41, A747 (1999)] for $D_{n c}, D_{I}$ and $D_{R}$ [C. C. Hegna, Phys. Plasmas 6, 3980 (1999); A. H. Glasser et al., Phys. Fluids 18, 875 (1975)], which are the bootstrap current driven term and the ideal and resistive interchange mode criterion, respectively. In addition to these components, the NIMROD routines calculate $\alpha_{s}-H$, a new correction to the PfirschSchlüter term. Finite parallel transport effects were added and a National Spherical Torus Experiment (NSTX) [M. Ono et al., Nucl. Fusion 40, 557 (2000)] equilibrium was analyzed. Another program takes the output of PEST-3 and allows the user to
\end{abstract}


specify the rational surface, island width, and amount of detail near the perturbed surface to visualize the total helical flux. The results of this work will determine the stability of NTMs in an spherical torus (ST) [Y.-K. M. Peng et al., Nucl. Fusion 26, 769 (1986)] plasma with greater accuracy than previously achieved. 


\section{Introduction and Motivation}

Neoclassical tearing modes (NTMs) can lead to disruption and loss of confinement [1]. The stability of this phenomenon in a Spherical Torus (ST) plasma [2] is insufficiently determined. Previous experimental analysis relied on the island evolution equation [3]

$$
\frac{\mathrm{d} w}{\mathrm{~d} t}=1.22 \frac{\eta_{n c}}{\mu_{0}}\left(\Delta^{\prime}+4.6 \frac{D_{n c}+D_{R}}{w}\right) .
$$

Here, $w$ is the full island width, $\eta_{n c}$ is the neoclassical resistivity, $\Delta^{\prime}$ is the jump in logarithmic derivative of $\psi$ over the rational surface, $D_{R}$ is the resistive interchange parameter (see Eq. 3), and $D_{n c}$ represents the bootstrap current contribution. $D_{n c}$ is destabilizing (positive) wherever $q^{\prime}$ and pressure derivative $p^{\prime}$ have opposite signs, where ' indicates a derivative with respect to $\psi$, the poloidal flux function labeling the magnetic surfaces. As pressure almost always decreases away from the magnetic axis and $q$ usually increases in a tokamak and ST, $D_{n c}$ is typically destabilizing. While $D_{R}$ is typically negative and therefore stabilizing, the resistive interchange effect is usually much smaller than the neoclassical tearing mode drive in conventional high aspect ratio tokamaks $\left(D_{n c} \gg D_{R}\right)$. However, at low aspect ratio, these two effects can become comparable [3]. This implies that through plasma shaping or current profile modification, it may be possible to use the beneficial effect of $D_{R}$ to make STs less susceptible to neoclassical tearing modes.

Originally formulated by Kotschenreuther et al. [4], this theory has recently been rederived for arbitrary aspect ratio and $\beta[5]$, thus adding applicability to ST plasmas. This new more general formula is:

$$
\frac{k_{0}}{\eta *} \frac{\mathrm{d} w}{\mathrm{~d} t}=\Delta *+\frac{k_{1}}{w}\left(D_{n c}+\frac{D_{R}}{\alpha_{s}-H}\right) .
$$

In this case, $\eta *$ is the resistive diffusion coefficient in flux space, and $w$ is in the same units 
as $\psi$. Other components of $(2)$ are $[5,6]$

$$
\begin{aligned}
& \Delta *=\Delta^{\prime}|w / 2|^{-2 \alpha_{l}} \sqrt{-4 D_{I}} \\
& \alpha_{l, s}=1 / 2 \mp \sqrt{-D_{I}} \\
& D_{R}=D_{I}+(H-1 / 2)^{2} \\
& D_{I}=E+F+H-1 / 4
\end{aligned}
$$

It should be noted that as $\beta$ goes to zero, $\Delta *$ reduces to the original result of $\Delta^{\prime}$. In these equations, $E, F$, and $H$ are functions of magnetic field, pressure, safety factor $q$, and $\psi$, first derived by Glasser, Greene, and Johnson [6]. $k_{0}$ and $k_{1}$ are constants depending on the pressure and flux function profiles. (Exact expressions for $D_{n c}, k_{0}, k_{1}$, and $\eta *$ can be found in Ref. [5], along with a more accurate definition of $\Delta^{\prime}$. Useful expressions for $D_{I}$ and $H$ are found in Ref. [7].) The $\alpha_{s}-H$ factor, which asymptotes to unity as $\beta$ tends to zero, diminishes the stabilizing effect of the Pfirsch-Schlüter term, and calls back into question how stable ST plasmas truly are to NTMs.

Another effect of some concern is coupling between singular layers with a common toroidal mode number. Focusing on the intermediate regime of the ideal magnetohydrodynamic (MHD) instability spectrum, modes with wavelengths short compared with machine size but long compared with a gyroradius, Dewar and Glasser employ a modified version of WKB theory to examine this coupling in Ref. [8]. A numerical model for a tokamak with a strong toroidal ripple shows coupling to be very weak for low-order modes, though this is not necessarily the case in an ST geometry. Thus both single and multiple helicity code packages are eventually desirable, however this work focuses on the single helicity case.

A program, ANTYEM, calling PEST-3 [9] and routines from NIMROD [10] has been developed in order to obtain a more accurate calculation of the right hand side (RHS) of Equation 2. PEST-3, a linear MHD stability code, is used to compute $\Delta^{\prime}$, and the NIMROD routines, 
specifically FLUXGRID, calculate $D_{I}, D_{R}, D_{n c}, \alpha_{l, s}$, and $H$. FLUXGRID reads in equilibrium data and performs the mappings required to have the data on a specified $\mathrm{R}(\psi, \theta)$, $\mathrm{Z}(\psi, \theta)$ grid while maintaining numerical accuracy.

Also, a new visualization program, HELUX, takes the output of PEST-3 and allows the user to specify island width, rational surface, and amount of detail near the perturbed surface to view the total helical flux as a contour plot.

\section{Derivations and Normalizations}

For completeness, the necessary steps to derive the final expressions of growth rate and helical flux used in the above programs are listed here.

\section{II.A Normalizations}

For ease of use, $w, \Delta *$, and $\mathrm{d} w / \mathrm{d} t$ have been normalized to the absolute value of the total flux, $\psi_{t}$, in the above programs as follows:

$$
\begin{aligned}
& \psi_{t}=|\psi(a)-\psi(0)| \\
& \bar{w}=\frac{w}{\psi_{t}} \\
& \bar{\Delta}=\psi_{t} \Delta *=\psi_{t}^{2 \mu} \Delta^{\prime}\left(\frac{\bar{w}}{2}\right)^{-1+2 \mu} 2 \mu \\
& \frac{\mathrm{d} \bar{w}}{\mathrm{~d} \tau}=\psi_{t} \frac{k_{0}}{\eta *} \frac{\mathrm{d} w}{\mathrm{~d} t}
\end{aligned}
$$

where $a$ labels the last closed flux surface, $\psi(0)$ is the flux on the magnetic axis, and $\mu=$ $\sqrt{-D_{I}}$. 


\section{II.B Helical flux for HELUX}

The helical flux function is a magnetic field line invariant that labels the flux surfaces in the presence of an isolated magnetic island chain. It is defined as:

$$
\psi_{h}=\frac{1}{2 \pi} \int_{\psi_{s}}^{\psi} \oint d \psi d \theta \mathcal{J} \nabla \alpha \cdot \mathbf{B}
$$

where $\mathcal{J}$ is the Jacobian

$$
\mathcal{J}=\frac{1}{(\nabla \psi \times \nabla \theta) \cdot \nabla \alpha}
$$

and angle coordinate

$$
\alpha=\zeta-q_{s} \theta
$$

corresponds to the helical angle of the resonant perturbation we will introduce [5]. $q_{s}$ is the $q=m / n$ of the chosen rational surface, $\zeta$ is the toroidal angle, and $\theta$ the poloidal angle. It should also be noted that $q$ is solely a function of $\psi$, and likewise $q_{s}$ corresponds to $\psi_{s}$.

The equilibrium magnetic field is then given by

$$
\mathbf{B}_{\mathbf{0}}=\nabla \psi \times \nabla(q \theta-\zeta)=\nabla \psi \times\left[\left(q-q_{s}\right) \nabla \theta-\nabla \alpha\right]
$$

We consider a magnetic perturbation of the form

$$
\tilde{\mathbf{B}}=\nabla \theta \times \nabla \tilde{A}
$$

where the perturbed vector potential magnitude $\tilde{A}$ can be expressed as 


$$
\tilde{A}=\sum_{m} A_{m}(\psi) e^{i(m \theta-n \zeta)}=\sum_{m} A_{m}(\psi) e^{i\left[\left(m-m_{s}\right) \theta-n \alpha\right]} .
$$

This describes a symmetry-breaking "radial" magnetic perturbation at the rational surface $q_{s}$. The total magnetic field may now be written as

$$
\mathbf{B}=\nabla \psi \times\left[\left(q-q_{s}\right) \nabla \theta-\nabla \alpha\right]+\nabla \theta \times \nabla \tilde{A}
$$

As a field line invariant, the helical flux function must obey the equation

$$
\mathbf{B} \cdot \nabla \psi_{h}=\frac{1}{\mathcal{J}}\left[\frac{\partial \psi_{h}}{\partial \theta}+\left(q-q_{s}-\frac{\partial \tilde{A}}{\partial \psi}\right) \frac{\partial \psi_{h}}{\partial \alpha}+\frac{\partial \tilde{A}}{\partial \alpha} \frac{\partial \psi_{h}}{\partial \psi}\right]=0
$$

Integrating this equation over $\theta$ eliminates the first term as well as the $\theta$ dependence of the other terms, setting $m=m_{s}$. To an arbitrary constant, the real solution to this equation is then

$$
\psi_{h}=\int_{\psi_{s}}^{\psi} d \psi\left(q-q_{s}\right)-A_{m_{s}} \cos (n \alpha) .
$$

This is the general expression for helical flux throughout an initially axisymmetric toroidal plasma. We will now specifically examine the area outside the island region, where ideal MHD theory is assumed to hold, and explicity use Equation 5. The perturbed field here is

$$
\tilde{\mathbf{B}}=\nabla \times\left(\boldsymbol{\xi} \times \mathbf{B}_{\mathbf{0}}\right)
$$

where $\boldsymbol{\xi}$, the perturbed displacement, can be written

$$
\boldsymbol{\xi}=\sum_{m} \boldsymbol{\xi}_{m}(\psi) e^{i(m \theta-n \zeta)}=\sum_{m} \boldsymbol{\xi}_{m}(\psi) e^{i\left[\left(m-m_{s}\right) \theta-n \alpha\right]} \quad \xi^{z}=\boldsymbol{\xi} \cdot \nabla z
$$


The helical flux may then be written as

$$
\psi_{h}=\psi_{h 0}+\tilde{\psi}_{h}
$$

where $\psi_{h 0}$ corresponds to the equilibrium helical flux associated with $\mathbf{B}_{\mathbf{0}}$ and $\tilde{\psi}_{h}$ is the perturbed helical flux from $\tilde{\mathbf{B}}$. It is then found that

$$
\nabla \alpha \cdot \mathbf{B}_{\mathbf{0}}=\mathcal{J}^{-1}\left(q-q_{s}\right)
$$

and so

$$
\psi_{h 0}=\int_{\psi_{s}}^{\psi} d \psi\left(q-q_{s}\right)
$$

For the perturbed part

$$
\begin{gathered}
\mathcal{J} \nabla \alpha \cdot \tilde{\mathbf{B}}=\mathcal{J} \nabla \cdot\left[\left(\boldsymbol{\xi} \times \mathbf{B}_{\mathbf{0}}\right) \times \nabla \alpha\right]+\mathcal{J}\left(\boldsymbol{\xi} \times \mathbf{B}_{\mathbf{0}}\right) \cdot \nabla \times \nabla \boldsymbol{\alpha} \\
=\mathcal{J} \nabla \cdot\left[\xi^{\alpha} \mathbf{B}_{\mathbf{0}}-\mathcal{J}^{-1}\left(q-q_{s}\right) \boldsymbol{\xi}\right] \\
=\frac{\partial}{\partial \psi}\left[\left(q_{s}-q\right) \xi^{\psi}\right]+\frac{\partial}{\partial \theta}\left[\xi^{\alpha}+\left(q_{s}-q\right) \xi^{\theta}\right]+\frac{\partial}{\partial \alpha}[0] .
\end{gathered}
$$

Now, because $m-m_{s}$ is an integer, and for $m=m_{s}$, the dependence of $\boldsymbol{\xi}$ on $\theta$ through $\left(m-m_{s}\right) \theta$ disappears

$$
\oint d \theta \frac{\partial \boldsymbol{\xi}}{\partial \theta}=0
$$

So 


$$
\tilde{\psi}_{h}=\frac{1}{2 \pi} \int_{\psi_{s}}^{\psi} \oint d \psi d \theta \frac{\partial}{\partial \psi}\left[\left(q_{s}-q\right) \xi^{\psi}\right]=\left(q_{s}-q\right) \xi_{m_{s}}^{\psi} e^{-i n \alpha}
$$

and

$$
\psi_{h}=\psi_{h 0}+A \cos (n \alpha) \quad A=-A_{m_{s}}=\left(q_{s}-q\right) \xi_{m_{s}}^{\psi} .
$$

(As only the $\psi$ component of $\boldsymbol{\xi}$ is relevent, the superscript of $\xi_{m_{s}}$ will be dropped from here on.) Now, approaching a rational surface but still outside the island [9]

$$
\xi_{m_{s}} \approx C x^{-\frac{1}{2}-\mu}
$$

where $C$ is an arbitrary constant determined by the island width and $x=\psi-\psi_{s}$. Assuming small islands, or $\bar{w} \ll 1$,

$$
q \approx q_{s}-q_{s}^{\prime} x \quad \psi_{h 0} \approx \frac{q_{s}^{\prime}}{2} x^{2}
$$

At the separatrix, $\psi_{h}\left(x=\frac{w}{2}, n \alpha=\pi\right)=\psi_{h}(0,0)$, so

$$
\frac{q_{s}^{\prime}}{2} \frac{w^{2}}{4}-A_{0} \approx A_{0} \quad \Rightarrow \quad A_{0} \approx q_{s}^{\prime} \frac{w^{2}}{16}
$$

where $A_{0}$ is the value of $A$ at the boundary. We also know from Eqs. 22 and 23 that

$$
A \approx-q_{s}^{\prime} x C x^{-\frac{1}{2}-\mu}
$$

Due to pressure flattening over the island, at the separatrix $D_{I} \rightarrow-1 / 4$, so $\mu \rightarrow 1 / 2[9]$. Thus a singularity at the rational surface is avoided, as 


$$
A \rightarrow A_{0} \approx-q_{s}^{\prime} C
$$

and comparing with Eq. 25,

$$
C=-\frac{w^{2}}{16}
$$

so, rewriting $\xi_{m_{s}}$ as $C \hat{\xi}_{m_{s}}$, outside the island

$$
\psi_{h}=\int_{\psi_{s}}^{\psi} d \psi\left(q-q_{s}\right)+\frac{w^{2}}{16}\left(q-q_{s}\right) \hat{\xi}_{m_{s}} \cos (n \alpha) .
$$

Inside the island, for the purposes of visualization HELUX assumes $A$ in Eq. 22 equals constant $A_{0}$, though, particularly for large $\beta$, it is likely to be a more complicated function.

\section{Transport Effects}

Due to the finite parallel thermal conductivity of the plasma, particles may diffuse across an island on the same time scale that they follow the field line around it, thus enhancing the pressure gradient over the island [11]. This is a stabilizing effect not covered in Hegna's theory, and it modifies the bootstrap term's $1 / w$ on the RHS of Eq. 2 to $w /\left(w^{2}+w_{d}^{2}\right)$. In its original formulation by Fitzpatrick for large aspect ratio and low $\beta$, taking $w$ in units of length [11]

$$
w_{d}=1.8 r_{s} \sqrt{\frac{8}{\epsilon_{s} s_{s} n}}\left(\frac{\chi_{\perp}}{\chi_{\|}}\right)^{1 / 4}
$$

where $r_{s}$ is the minor radius of the rational surface under consideration, $\epsilon_{s}=r_{s} / R_{0}, s_{s}$ is local magnetic shear $\left(r \partial_{r} q / q\right)_{r_{s}}, n$ is the toroidal mode number, and $\chi_{\perp}$ and $\chi_{\|}$are the 
perpendicular and parallel thermal conductivities, respectively. Ref. [11] also gives general forms

$$
\chi_{\perp} \sim \frac{a^{2}}{6 \tau_{e}} \quad \text { and } \quad \chi_{\|} \sim v_{t e} \lambda_{\|}
$$

where $a$ is the minor radius of the outermost flux surface, $\tau_{e}$ is the global energy confinement time, $v_{t e}$ is the electron thermal velocity, and $\lambda_{\|} \equiv r_{s}^{2} /\left(\epsilon_{s} s_{s} n w_{d}\right)[12]$ is the parallel wavelength of the helical perturbations. Eq. 30 may then be rewritten as:

$$
w_{d}=1.8 \sqrt{8} r_{s}\left(\frac{w}{r_{s}} \frac{1}{s_{s} \epsilon_{s} n} \frac{\chi_{\perp}}{r_{s} v_{t e}}\right)^{1 / 4} .
$$

To apply the above expression to this paper's definition of $w$, and take into account the more shaped nature of ST flux surfaces, it is assumed that $\psi$ is roughly proportional to $r^{2}$ in any direction away from the magnetic axis. Thus, the following substitutions are made:

$$
\begin{aligned}
& r_{s} \text { in front } \rightarrow 2 \psi_{s} \\
& s_{s} \rightarrow 2 \bar{s}_{s}, \quad \bar{s}_{s}=\left(\psi q^{\prime} / q\right)_{\psi_{s}} \\
& \frac{w}{r_{s}} \rightarrow \frac{w}{2 \psi_{s}}
\end{aligned}
$$

The remaining $r_{s}$ in the denominator of Eq. 32 is estimated as the geometric mean of $a_{s}$ and $b_{s}$, or $\sqrt{a_{s} b_{s}}$, where these two quantities are half the maximum horizontal and vertical displacement of surface $\psi_{s}$. The resulting expression may then be normalized with a division by $\psi_{t}$, yielding:

$$
\bar{w}_{d}=7.2\left(\frac{\psi_{s}}{\psi_{t}}\right)^{3 / 4}\left(\bar{w} \frac{1}{\bar{s}_{s} \epsilon_{s} n} \frac{\chi_{\perp}}{r_{s} v_{t e}}\right)^{1 / 4}
$$


Finally, multiplying Eq. 2 through by $\psi_{t}$ and including transport effects as per Refs. [11] and [13], it may be rewritten in completely dimensionless form as follows:

$$
\frac{\mathrm{d} \bar{w}}{\mathrm{~d} \tau}=\bar{\Delta}+k_{1}\left(\frac{\bar{w}}{\bar{w}^{2}+\bar{w}_{d}^{2}} D_{n c}+\frac{1}{\sqrt{\bar{w}^{2}+0.65 \bar{w}_{d}^{2}}} \frac{D_{R}}{\alpha_{s}-H}\right) .
$$

Finite transport effects tend to eliminate the neoclassical drive as the island width becomes smaller than $w_{d}$. Recent work by Lütjens, Luciani, and Garbet suggests that the resistive term scales differently than the neoclassical term when the island width is smaller than the critical width [13]. Namely, the interchange term asymptotes to a non-zero value as the island width goes to zero. When the island width greatly exceeds $w_{d}$, Eq. 35 is consistent with Eq. 2.

\section{Demonstration}

\section{IV.A ANTYEM}

To demonstrate the function of these programs on a large ST, National Spherical Torus Experiment (NSTX) [14] shot \#103698 at 192ms is analyzed. It has a $q$ on axis of 1.08 and a $\beta_{t}$ of $16.1 \%$. Figure 1 shows growth rate $\mathrm{d} \bar{w} / \mathrm{d} \tau$, as given in Eq. 35, as a function of $\bar{w}$ for the $q_{s}=3 / 2$ and $q_{s}=4 / 3$ rational surfaces. Both of these curves are negative everywhere, indicating that these modes are stable. $D_{n c}+D_{R} /\left(\alpha_{s}-H\right)$ is greater than zero, meaning the neoclassical tearing drive overcomes the stabilizing Pfirsch-Schlüter term, however the presence of this term reduces the drive enough that transport effects can stabilize the island.

A notable clarification is that precise knowledge of the numerical factor $k_{1}$ is important in quantitative predictions of neoclassical tearing mode stability. Because the analytic theory is based upon asymptotic arguments, it is difficult to predict precise numerical factors of order unity. Therefore $k_{1}$ needs to be experimentally determined. The value of $k_{1}=1.7$ has been 
used, along with (less critical) values $T_{e}=400 \mathrm{eV}$ and $\tau_{e}=30 \mathrm{~ms}$. This $k_{1}$ was determined by matching to the observed island saturation width in Tokamak Fusion Test Reactor (TFTR) discharges [15]. The conducting wall in PEST-3 was set as $20 \%$ of the midplane minor radius away from the outermost flux surface.

It is also of note that the calculated $\Delta^{\prime}$ in these cases does not follow the approximation used in previous analysis of high aspect ratio, low $\beta$ tokamaks, that is $r_{s} \Delta^{\prime} \approx-2 m$. [3] If $r_{s}$ is appropriately averaged over the corresponding rational surface, becoming $\left\langle r_{s}\right\rangle$ with units of $\left[\psi^{2 \mu}\right]$, then for the $3 / 2$ surface $\left\langle r_{s}\right\rangle \Delta^{\prime}$ is calculated to be -9.03 , and -10.98 on the $4 / 3$ surface. Another important topic is the means by which $\Delta^{\prime}$ was determined from the output of PEST-3, which is covered in the Appendix.

\section{IV.B HELUX}

Figure 2 shows a visualization of $\psi_{h}$ as defined in Eq. 29 for rational surfaces $3 / 2$ and $4 / 3$ on NSTX shot \#103698, 192ms with imposed island widths $\bar{w}=10 \%$ and $\bar{w}=15 \%$ respectively. HELUX allows one to specify any $\bar{w}$ in any toroidal equilibrium for any rational surface one wishes, though it should be noted that the larger $\bar{w}$ is, the less accurate Eq. 29 is likely to be.

\section{Summary}

Using the best available theoretical and computational models, a new tool has been created which determines neoclassical tearing mode growth rates more accurately than previously achieved for high $\beta$ and low aspect ratio. An NSTX equilibrium has been analyzed, and the stabilizing effects of the Pfirsch-Schlüter current and finite parallel conductivity were determined to be competitive with the neoclassical tearing drive. Also, using an expression for helical flux derived in Section II.B, another tool has been created which allows one to 
visualize magnetic islands of any size for any toroidal equilibrium. Finally, the convergence properties of PEST-3's $\Delta^{\prime}$ have been examined. The numerical computation of $\Delta^{\prime}$ was shown to be significantly different from the large aspect ratio analytic prediction in NSTX's parameter regime. The accuracy of this value can be critically important to determining overall stability to NTMs.

Recommended future work includes a more general theory for the effects of finite parallel thermal conductivity and either derivation of a more explicit formula for $k_{1}$ or experimental determination of the constant. Also, a more complete theory would include a more accurate expression for helical flux within a neoclassical island for arbitrary $\beta$.

\section{Acknowledgements}

The authors wish to thank Bill Davis for his helpful assistance with visualization in IDL. This research is supported by the U.S. Department of Energy under contract No. DE-AC02CHO3073.

\section{Appendix: $\Delta^{\prime}$ Convergence with PEST-3}

For the computation of $\Delta^{\prime}$, the latest version of the PEST-3 code has been used. Recently, PEST-3 underwent significant changes to improve its ease of use. The code can now be invoked as a UNIX command with input parameters specified as command arguments. More importantly, PEST-3 now has the ability to interface directly to various equilibrium file formats and codes, including EFIT, TRANSP, JSOLVER, ESC, and CHEASE. Also, the

code can now connect directly to existing MDSPlus databases. This version is freely available from http://w3.pppl.gov/NTCC.

The scheme used in PEST-3 to determine $\Delta^{\prime}$ and other matching data is based on computing 
the difference between two energies, one involving the small solution and the other the large solution [9]. Since these two energy components often are comparable but of opposite sign, care should be taken to ensure that the computed $\Delta^{\prime}$ is accurate. In particular, highly accurate equilibria are required since the large solution depends on higher order derivatives of equilibrium profiles. In general, $\Delta^{\prime}$ calculations tend to be more sensitive to equilibrium inaccuracies than ideal stability calculations for this reason.

PEST-3 uses linear (hat) functions, or $\wedge$, as radial finite elements. To ensure that $\Delta^{\prime}$ converges quadratically in the inverse number of radial elements $\mathrm{mm}^{-1}$, the mesh must be packed in the neighborhood of rational surfaces where the numerical (small) solution $\sim\left|\psi-\psi_{s}\right|^{-1 / 2+\mu}$ is singular. It can be shown [16] that the optimal node distribution near $\psi_{s}$ for $\mu \neq 1 / 2$ is $\psi_{i}=\psi_{s}+\left|\frac{i-i_{s}}{m m}\right|^{1 / \mu}$ where $i$ is the node index and $i_{s}$ the index of the node at the rational surface, while for $\mu=1 / 2$ a uniform mesh is appropriate. Although we have dealt here with a single helicity mode, the code PEST-3 allows for several resonant surfaces with mesh packing applied (automatically) on each rational surface where reconnection occurs. The treatment of multi-resonant surfaces could be of importance when modes with different helicity have comparable rotation speed, which can lead to mode locking. In this study, $\mathrm{mm}$ was typically varied from 70 to 299 . The final, chosen $\Delta^{\prime}$ was obtained after extrapolating to infinite $m m$ and assuming convergence with $m^{-2}$. Figure 3 shows a convergence plot for the 3/2 mode of NSTX shot \#103698 at $192 \mathrm{~ms}$; the extrapolated value is $\psi_{s}^{2 \mu} \Delta^{\prime}=-3.38$.

For the 2/1 mode in NSTX, however, we found that the convergence often failed to yield a unique, infinite resolution $\Delta^{\prime}$ because the mode was close to the ideal marginal stability point, as verified by PEST 2 [17] for the above equilibrium. Recall that at marginal stability, $\Delta^{\prime}$ transitions through a pole (positive on the stable side, negative on the unstable side) and is thus ill defined. In this case it would be preferable to work using $1 / \Delta^{\prime}$ matching data, which is well behaved across the ideal marginal point. For plasmas on the stable side of the ideal marginal stability point, $\Delta^{\prime}$ tends to be large and positive as is often observed for the 
2/1 mode. This should drive the mode unstable. We can only speculate why higher order modes $(3 / 2,3 / 4$ etc. $)$ tend to be more often observed experimentally than the $2 / 1$. One possibility is that the asymptotic matching method does not apply for large $\Delta^{\prime}$. Another possibility is that quasi-linear stabilization terms play an important role [18]. These issues can only be resolved by performing nonlinear NTM simulations. 


\section{References}

[1] D. A. Gates and R. LaHaye, Nucl. Fusion 39, 2251 (1999).

[2] Y.-K. M. Peng and D. J. Strickler, Nucl. Fusion 26, 769 (1986).

[3] S. E. Kruger, C. C. Hegna, and J. D. Callen, Phys. Plasmas 5, 455 (1998).

[4] M. Kotschenreuther, R. D. Hazeltine, and P. J. Hazeltine, Phys. Fluids 28, 294 (1985).

[5] C. C. Hegna, Phys. Plasmas 6, 3980 (1999).

[6] A. H. Glasser, J. M. Greene, and J. L. Johnson, Phys. Fluids 18, 875 (1975).

[7] J. M. Greene, Comments on Modern Physics Part E 17, 389 (1997).

[8] R. L. Dewar and A. H. Glasser, Phys. Fluids 26, 3038 (1983).

[9] A. Pletzer, A. Bondeson, and R. L. Dewar, J. Comput. Phys. 115, 530 (1994).

[10] A. H. Glasser, C. R. Sovinec, R. A. Nebel, T. A. Gianakon, S. J. Plimpton, M. S. Chu, and D. D. Schnack, Plasma Phys. Controlled Fusion 41, A747 (1999).

[11] R. Fitzpatrick, Phys. Plasmas 2, 825 (1994).

[12] D. A. Gates, B. Lloyd, A. W. Morris, G. McArdle, M. O’Brien, M. Valovic, C. Warrick, H. Wilson, and Compass-D Team and ECRH Team, Nuclear Fusion 37, 1593 (1997).

[13] H. Lütjens, J.-F. Luciani, and X. Garbet, Phys. Plasmas 8, 4267 (2001).

[14] M. Ono, S. M. Kaye, and Y.-K. M. Peng et al., Nucl. Fusion 40, 557 (2000).

[15] Z. Chang, J. D. Callen, E. D. Fredrickson, R. V. Budny, C. C. Hegna, K. M. McGuire, M. C. Zarnstorff, and TFTR Group, Phys. Rev. Lett. 74, 4663 (1995).

[16] A. Pletzer and R. L. Dewar, J. Plasma Phys. 45, 427 (1991). 
[17] R. C. Grimm, R. L. Dewar, and J. Manickam, J. of Comput. Phys. 49, 94 (1983).

[18] A. Pletzer and F. W. Perkins, Phys. Plasmas 6, 1589 (1999). 
FIG. 1: $3 / 2$ and 4/3 modes, $\frac{\mathrm{d} \bar{w}}{\mathrm{~d} \tau}$ vs. $\bar{w}$ for NSTX shot \#103698 at $192 \mathrm{~ms}$, which has a $q$ on axis of 1.08 and $\beta_{t}$ of $16.1 \%$. These modes appear stable, pending a more accurate determination of constant $k_{1}$ in growth rate Equation 35 .

FIG. $2: 3 / 2, \bar{w}=10 \%$ and $4 / 3, \bar{w}=15 \%$ simulated island structures for NSTX shot \#103698 at $192 \mathrm{~ms}$.

FIG. 3: Convergence of normalized $\Delta^{\prime}$ for the $3 / 2$ mode vs. the inverse square of the number of radial finite elements for NSTX shot \#103698 at $192 \mathrm{~ms}$. 

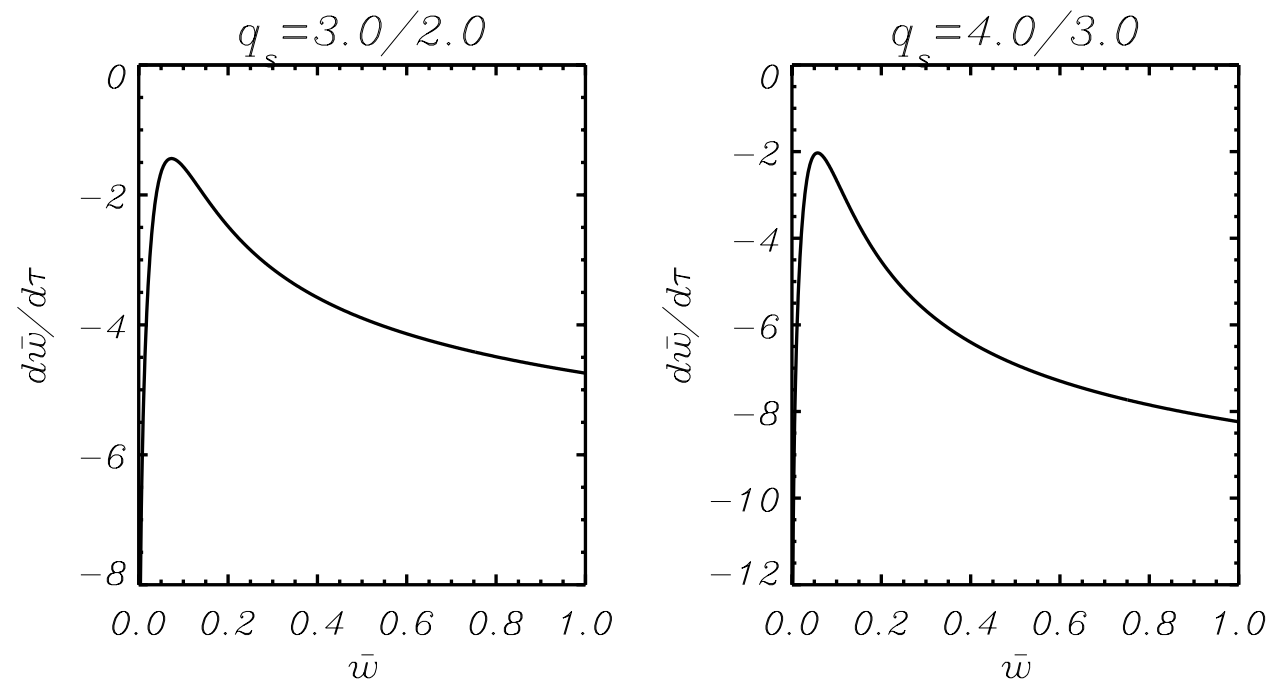

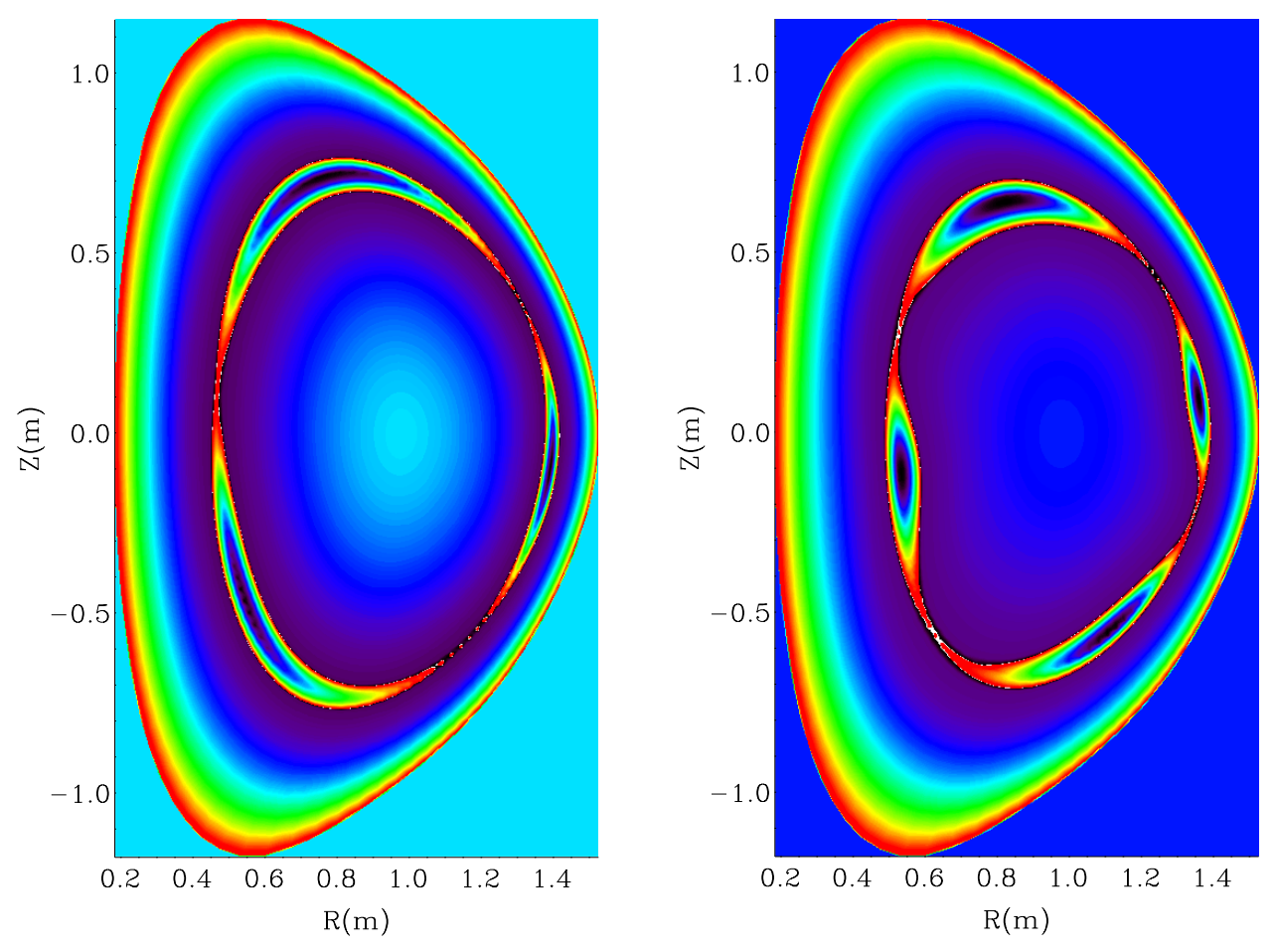


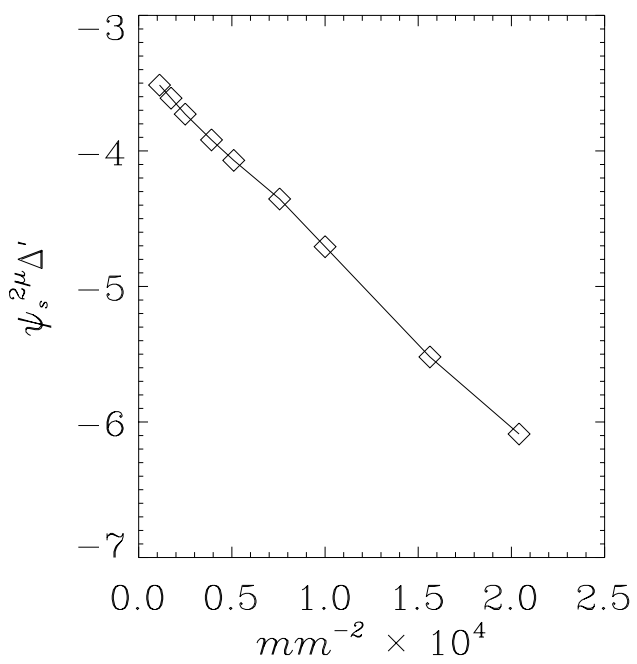




\section{External Distribution}

Plasma Research Laboratory, Australian National University, Australia

Professor I.R. J ones, Flinders University, Australia

Professor J oão Canalle, Instituto de Fisica DEQ/IF - UERJ , Brazil

Mr. Gerson O. Ludwig, Instituto Nacional de Pesquisas, Brazil

Dr. P.H. Sakanaka, Instituto Fisica, Brazil

The Librarian, Culham Laboratory, England

Library, R61, Rutherford Appleton Laboratory, England

Mrs. S.A. Hutchinson, JET Library, England

Professor M.N. Bussac, Ecole Polytechnique, France

Librarian, Max-Planck-Institut für Plasmaphysik, Germany

J olan Moldvai, Reports Library, MTA KFKI-ATKI, Hungary

Dr. P. Kaw, Institute for Plasma Research, India

Ms. P.J . Pathak, Librarian, Insitute for Plasma Research, India

Ms. Clelia De Palo, Associazione EURATOM-ENEA, I taly

Dr. G. Grosso, Instituto di Fisica del Plasma, Italy

Librarian, Naka Fusion Research Establishment, J AERI, J apan

Library, Plasma Physics Laboratory, Kyoto University, J apan

Research Information Center, National Institute for Fusion Science, J apan

Dr. O. Mitarai, Kyushu Tokai University, J apan

Library, Academia Sinica, Institute of Plasma Physics, People's Republic of China

Shih-Tung Tsai, Institute of Physics, Chinese Academy of Sciences, People's Republic of China

Dr. S. Mirnov, TRINITI, Troitsk, Russian Federation, Russia

Dr. V.S. Strelkov, Kurchatov Institute, Russian Federation, Russia

Professor Peter Lukac, Katedra Fyziky Plazmy MFF UK, Mlynska dolina F-2, Komenskeho Univerzita, SK-842 15 Bratislava, Slovakia

Dr. G.S. Lee, Korea Basic Science Institute, South Korea

Mr. Dennis Bruggink, Fusion Library, University of Wisconsin, USA

Institute for Plasma Research, University of Maryland, USA

Librarian, Fusion Energy Division, Oak Ridge National Laboratory, USA

Librarian, Institute of Fusion Studies, University of Texas, USA

Librarian, Magnetic Fusion Program, Lawrence Livermore National Laboratory, USA

Library, General Atomics, USA

Plasma Physics Group, Fusion Energy Research Program, University of California at San Diego, USA

Plasma Physics Library, Columbia University, USA

Alkesh Punjabi, Center for Fusion Research and Training, Hampton University, USA

Dr. W.M. Stacey, Fusion Research Center, Georgia Institute of Technology, USA

Dr. J ohn Willis, U.S. Department of Energy, Office of Fusion Energy Sciences, USA

Mr. Paul H. Wright, Indianapolis, Indiana, USA 
The Princeton Plasma Physics Laboratory is operated by Princeton University under contract with the U.S. Department of Energy.

\author{
Information Services \\ Princeton Plasma Physics Laboratory \\ P.O. Box 451 \\ Princeton, NJ 08543
}

Phone: 609-243-2750

Fax: 609-243-2751

e-mail: pppl_info@pppl.gov

Internet Address: http://www.pppl.gov 